\title{
Raspberry Pi Driven Flow-Injection System for Electrochemical Continuous Monitoring Platforms
}

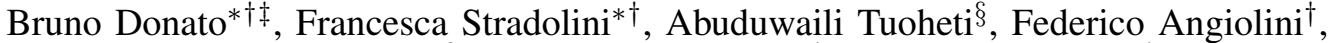 \\ Danilo Demarchi $^{\S}$, Giovanni De Micheli ${ }^{\dagger}$ and Sandro Carrara ${ }^{\dagger}$ \\ ${ }^{\dagger}$ Laboratory of Integrated Systems, Swiss Federal Institute of Technology - Lausanne (EPFL), Switzerland \\ $\ddagger$ Department of Information Engineering, Electronics and Telecommunications, Sapienza University of Rome, Italy \\ $\S$ Department of Electronics and Telecommunications, Politecnico di Torino, Italy \\ *Corresponding author: bruno.donato@epfl.ch, francesca.stradolini@epfl.ch
}

\begin{abstract}
The degree of interest in bio-sensing platforms brings to the forefront a corresponding need for effective testing of their capabilities. This necessity is even more crucial when examining the properties of a sensor for continuous monitoring of a concentration trend in time, before in vivo implementations. Moreover, in the framework of personalised medical practices, it is imperative to introduce a robust way to represent and parametrise the highly variable responses of human metabolism. The aim of this paper is to propose a novel solution for the design of an automatic flow-injection environment that can assess the performance of systems for continuous monitoring. The setup is also approved for successfully reproducing a paracetamol concentration trend in buffer solution.
\end{abstract}

\section{INTRODUCTION}

Up to now, the concept of continuous monitoring has been widely exploited in different medical applications for keeping under control endogenous [1], [2] and exogenous [3], [4] compound concentrations in patients' fluids. The need for drug monitoring services is widespread, especially in the context of personalised medicine to ensure the efficacy and the safety of a particular drug administration to a patient [5]. This holds particularly true for difficult-to-handle drugs, such as anaesthetics. Indeed, anaesthesia induction and maintenance rely on a right balance of three main compounds (anaesthetic, analgesic and muscle relaxant) to be injected in the patient's veins [6]. In similar practices, to provide the patient with the optimum drug dosage, it is fundamental to know the dissolution behaviour of the drug itself after it is administered [7]. This is known as the drug Pharmaco-Kinetic (PK) model, that reproduces the absorption, distribution, metabolism and excretion of the drug [8]. Electrochemical sensors are considered as a suitable technology to keep under control the drug concentration variations in the patient's fluid. They enable on-line drug monitoring since they do not need any electrode surface renewal while measuring and their selectivity can be assessed by adopting suitable sensing material so that they are adaptable for several different applications [9]. For applications such as anaesthesia, in which the system depends on estimations made on human body metabolic responses [10], it is very difficult to find an experimental procedure to test sensor performance. To reproduce the drug trend after administration, dissolution tests of the pharmaceutical dosages are required. These experiments are extremely laborious, if not automated since a large number of samples are needed. Therefore, automated dissolution systems are desirable to save time, to improve analytical reproducibility in sensor validation [7]. Various approaches have been proposed to assess automatic testing procedure [11], [12], [13]. Nevertheless, these dissolution set-ups do not reproduce the entire PK profile of the drug over a prolonged period of time [7]. To this aim, in this paper we want to present the design, the realisation and the validation of a flow-injection system able to reproduce in a given base-volume of analysed solution a drug concentration time-trend. The dilution process to reproduce PK trend of a drug is handled by a Raspberry Pi electronic board that strikes an injection pump charged with the drug. The concentration level to reach is evaluated by a PK mathematical model implemented in Matlab. The system has been validated empirically by using a dye and experimentally by sensing $\mathrm{pH}$ variations and by reproducing a paracetamol trend. We have chosen paracetamol to demonstrate the possibility to adopt the system in testing anaesthesia monitoring systems. Indeed, paracetamol is an analgesic and can be considered as a benchmark anaesthesia compound.

\section{MATERiAls AND Methods}

\section{A. Chemicals and Instrumentation}

Green food dye from Dèco Relief ${ }^{\mathrm{TM}}$ was used to empirically evaluate the dissolution system performances. For the analytical validation, hydrochloric acid $(\mathrm{HCl}) 0.01 \mathrm{M}$ and Paracetamol (APAP) are provided by Sigma-Aldrich ${ }^{\mathrm{TM}}$. APAP stock solution $30 \mathrm{mM}$ was prepared by dissolving 15 $\mathrm{mg}$ of powder in $3 \mathrm{ml}$ Phosphate Buffer Saline (PBS) (pH: 7.4, $10 \mathrm{mM}$ ). To realise and test the system we adopted:(i) Gilson ${ }^{\mathrm{TM}}$ Minipuls 3 peristaltic pump, (ii) Braintree Scientific Inc. ${ }^{\text {TM }}$ NE-1000 Programmable Single Syringe Pump [14], (iii) VWR and Stuart CB161 stirrers, (iv) VWR pH $1000 \mathrm{~L}$ pHenomenal $^{\mathrm{TM}} \mathrm{pH}$ meter, (v) Raspberry Pi (RPi) 3 Model B running Raspbian Jessie 4.4, (vi) Metrohm Autolab ${ }^{\text {TM}}$ PGSTAT 101. Further we used: Duran ${ }^{\mathrm{TM}} 1 \mathrm{~mL}$ volumetric flask class $\mathrm{A}$, $1 \mathrm{~mL}$ and $0.5 \mathrm{~mL}$ plastic syringes $(\oslash 4.699 \mathrm{~mm}$ for colorant and $\mathrm{pH}$ ), Tygon ${ }^{\mathrm{TM}} \mathrm{LMT}-55$ peristaltic pump tubing $(\oslash 0.38$ $\mathrm{mm})$ for APAP and $\mathrm{pH}$ tests, PVC tubing $(\oslash 1 \mathrm{~mm})$ for dye, PTFE (AWG) inner $\oslash 0.41 \mathrm{~mm}$ tubing connected with the syringe needle. Dropsens ${ }^{\mathrm{TM}} \mathrm{DS} 110$ Screen-Printed Electrodes (SPEs) are adopted as electrochemical cell for APAP detection. They host on their surface a carbon Working ( $\oslash 4 \mathrm{~mm})$, a silver Reference and a carbon Counter Electrode.

As softwares we used: (i) Qt Creator 3.2 with Qt 5.3 libraries for the TCP/IP server and syringe pump serial communication application on $\mathrm{RPi}$, (ii) Matlab ${ }^{\mathrm{TM}}$ for injection control and dose computation, (iii) Microsoft ${ }^{\mathrm{TM}}$ Excel for data analysis, (iv) Metrohm Autolab ${ }^{\mathrm{TM}}$ NOVA 10.1 for electrochemical measurements. Cyclic Voltammetry (CV) measurements were run for APAP sensing with a NOVA procedure applying voltages from $-0.1 \mathrm{~V}$ to $1.1 \mathrm{~V}$ with $0.1 \mathrm{~V} / \mathrm{s}$ scan-rate.

\section{B. Calibration Procedure}

Before starting any test session a procedure to calibrate the flow inside the specific peristaltic tubing adopted was run. 


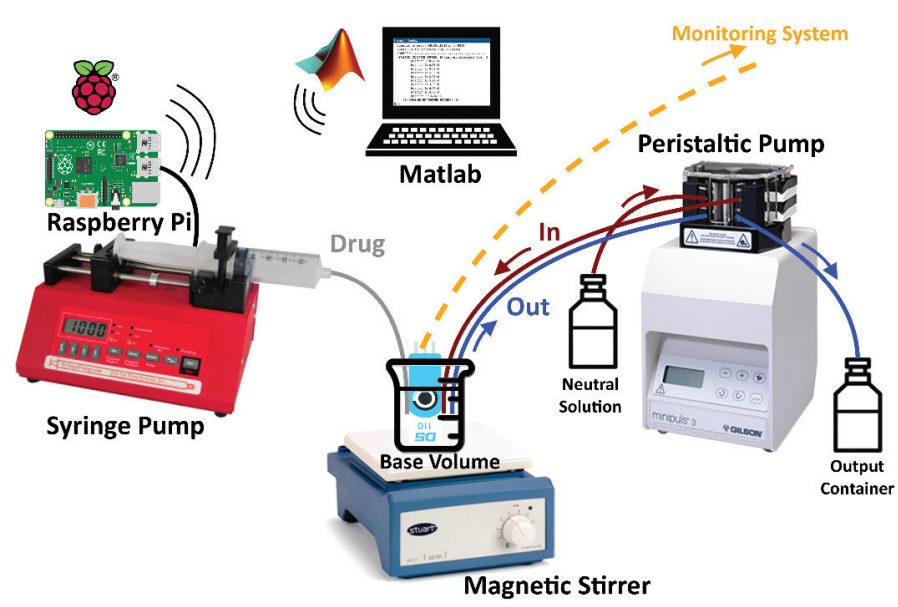

Fig. 1. Sketch of the flow-injection system: (from left to right) the RPi connected to the syringe pump, a PC running Matlab, the beaker containing the base-volume placed on the magnetic stirrer, and the peristaltic pump.

This allows us to establish the relation between pump rotor speed (Revolutions Per Minute - RPM) and tubing flow rate $(\mathrm{mL} / \mathrm{min})$. The calibration consists in setting the pump speed at different values and measuring the time for filling up a given volume (e.g. $1 \mathrm{~mL})$. A dedicated Excel workbook was realised to insert timings and pump speeds so that the flow rate $(\mathrm{mL} / \mathrm{min})$ corresponding to each pair of values is automatically calculated. Further, calibration lines are evaluated by simple linear regression of the flow rates. Indeed, the speed-flow relation is linear [15].

\section{SySTEM CONFIGURATION}

The configuration of the flow-injection system is shown in Fig. 1. We can consider its structure as divided in two main parts with respect to their functionality: the part including the syringe that is dedicated to increasing the drug concentration and the other part consisting of the peristaltic pump for decreasing it. The syringe pump is programmed to push the plunger of the syringe filled with the administered drug by means of a moving plate. The peristaltic pump withdraws the solution from the base-volume beaker and fills it with the neutral solution. The In/Out flows of the base-volume beaker are calibrated to maintain its volume constant. These two system sections are running in parallel in a synchronised way to reproduce the drug concentration trend in the base-volume. In other words, while the peristaltic pump continuously dilutes the base-volume with a neutral solution, the syringe pump injects the right amount of the drug to achieve the target concentration level. The peristaltic pump is calibrated and set at a fixed constant flow rate before starting each test. The RPi is the central unit of the system that connects the two parts making them interact. Indeed, the syringe pump is physically connected to the RPi through a USB port. The RPi communicates with Matlab, running on the PC, which calculates the subsequent injection doses relative to the previous concentration and the drug trend to reproduce. Finally, the RPi sends the evaluated injection value to the syringe pump. As soon as the syringe pump receives a command, an acknowledgement message is sent.

\section{A. Raspberry Pi Integration}

$\mathrm{RPi}$ is adopted as link between the Matlab script and the syringe pump. Indeed, we wanted to exploit its main advantages

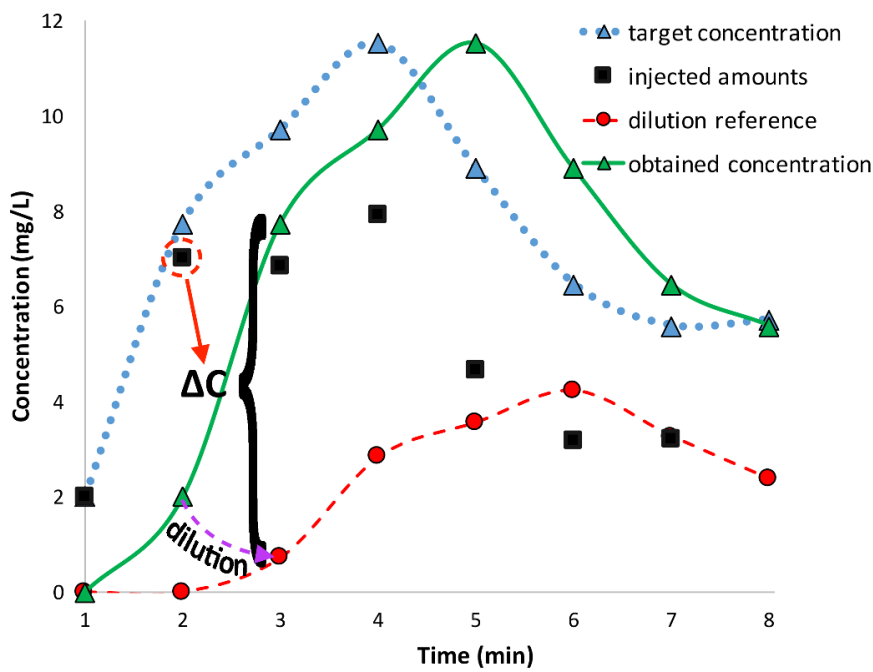

Fig. 2. Example of the injection dose calculation (injected amounts) applied to a sample target concentration trend (target concentration). The dilution prevision is determined considering a flow rate of $1 \mathrm{~mL} / \mathrm{min}$ in a $1 \mathrm{~mL}$ basevolume. Differences among the plots exaggerated for readability.

of portability, low-cost, and variety of configurable interfaces, among which USB ports and a Wi-Fi module. A light console application to handle the TCP/IP and serial communications is developed with the Qt Creator libraries. Thanks to this, data are sent to the RPi from Matlab, through WLAN, and transformed into serial commands for the pump. Moreover, due to its small dimensions we can use the RPi-based system also in restricted space by controlling it remotely. This is useful when toxic drug, e.g. anaesthetics, are involved since tests are performed in restricted and controlled environment.

Final aim of our project is to move the driving intelligence of the system into the RPi and remove the need for the PC to enhance and guarantee the system portability.

\section{B. Communication Protocols}

Two communication protocols are adopted: one to send data from $\mathrm{PC}$ to the $\mathrm{RPi}$, and the other to send commands from the RPi to the syringe pump. The first relies on TCP/IP since the RPi application uses the Wi-Fi module to create a WLAN that the PC can access as client. The second is RS-232 between RPi USB port and the syringe pump RJ-11 socket. The physical connection is realised using a custom adaptor.

\section{Syringe Pump Configuration}

The NE-1000 syringe pump works both in a manual or programmed mode through serial communication. Main setup parameters to be configured are: pumping rate, target volume, and syringe diameter. From these parameters, the pump automatically evaluates the injection time and regulates the plunger speed accordingly. The pump can be programmed to withdraw and inject liquids in different pumping phases. Each phase can be characterised by a different pumping rate or target volume. Through RPi we set the pump to perform direct injection every time it receives a new injection command. The injection is carried out by pushing uniformly the syringe plunger that injects constantly an amount of drug to reproduce a linear increase of drug concentration trend. 


\section{Injection Dose Calculation}

To calculate the right injection values for reproducing a concentration time-trend we need to consider the continuous dilution of the base-volume, executed by peristaltic flow at a constant and fixed speed. The relation between dilution and flow is expressed by (1) that determines how an initial concentration $\left[C_{i}\right]$ in a volume $\mathrm{V}$ decreases in time $\left(t_{f i}=t_{f}-t_{i}\right)$, while diluting with a flow rate Q [16].

$$
\left[C_{f}\right]=\frac{\left[C_{i}\right]}{e^{\frac{Q \cdot\left(t_{f}-t_{i}\right)}{V}}}
$$

where $\left[C_{f}\right]$ is the final concentration.

We can simplify (1) by expressing $\frac{\left[C_{f}\right]}{\left[C_{i}\right]}$ as a unique coefficient $\delta\left(t_{f i}\right)$. If we consider a continuous dilution $t_{f i}$ becomes an intermediate time $t_{m}$ between two consecutive measurements $\left(t_{n+1}-t_{n}\right)$. Therefore, the needed subsequent dose to reproduce the target time-trend of the drug concentration $\left(\left[C_{t}\right]\right)$ is calculated from (2) before every injection.

$$
\left[C_{\text {dose }}\right]= \begin{cases}{\left[C_{t}\right]-\left(\left[C_{n}\right] \cdot \delta\left(t_{m}\right)\right),} & {\left[C_{t}\right] \geq\left[C_{n}\right] \cdot \delta\left(t_{m}\right)} \\ 0, & {\left[C_{t}\right]<\left[C_{n}\right] \cdot \delta\left(t_{m}\right)}\end{cases}
$$

where $\left[C_{n}\right]$ is the concentration at $t=t_{n}, t_{m}=t_{n+1}-t_{n}$, and $\left[C_{\text {dose }}\right]$ is the required concentration to be injected.

Fig. 2 shows the concept of injection evaluation implemented in Matlab. The blue dotted line (target concentration) refers to the time-trend to reproduce, while the green line (obtained concentration) keeps track of the actual concentration in the basevolume. Considering the difference $\Delta \mathrm{C}$ between the diluted prevision of current concentration and target concentration, the new dose is evaluated and injected at the time $t_{n}$. Indeed, the dose $\left(\left[C_{\text {dose }}\right]\right.$ in (2)) calculation relies on the dynamic dilution continuously performed by the peristaltic pump. The algorithm predicts the drug concentration at the time $t_{n+1}$ if no further drug is injected, red dashed line in Fig. 2 (dilution prevision). The black squares in the figure (injected amounts) show all the concentration values to be administered. Before sending these amounts to the syringe pump, a previous conversion in volume unit is needed. This conversion depends on the concentration of the drug stock solution. The converted values are sent to the RPi that notifies the pump. The pump continuously infuses each volume in a time $t_{m}$ to ensure a smooth time-trend.

\section{RESUlts AND Discussion}

We scheduled three different tests for system validation. Firstly, we empirically tested the dilution ability of the system. Secondly, we challenged the system with a fast rising concentration followed by a decreasing of the concentration until a steady-state phase. Finally, we used the system to reproduce an increasing and decreasing trend for a real drug administration, the analgesic APAP.

\section{A. Test Results}

1) Dye Test: For the first test we injected green food dye in water as neutral solution. We programmed a set of dye injections in a $1 \mathrm{~mL}$ base-volume, with a peristaltic flow rate of $1 \mathrm{~mL} / \mathrm{min}$. The dosage was scheduled to linearly increase in time the concentration in the base-volume and then decrease it exponentially while diluting. The test was performed under stirring condition (level 9 of the CB161 stirrer). The visual results of the test are shown in Fig. 3. With this test we were able to see in the colour tone change how the system can reproduce a linear increase and an exponential decrease in concentration.

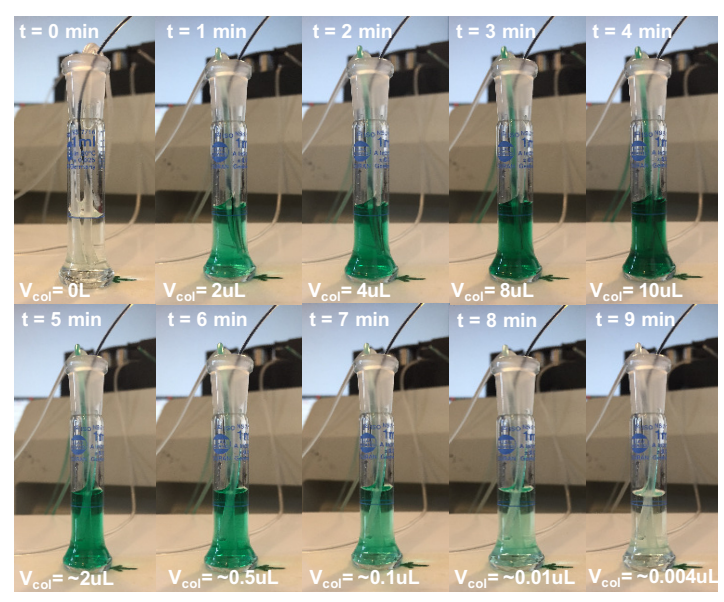

Fig. 3. Dye test: $V_{c o l}$ is the dye volume in the base-volume beaker. In the upper part these values are the dye injections.

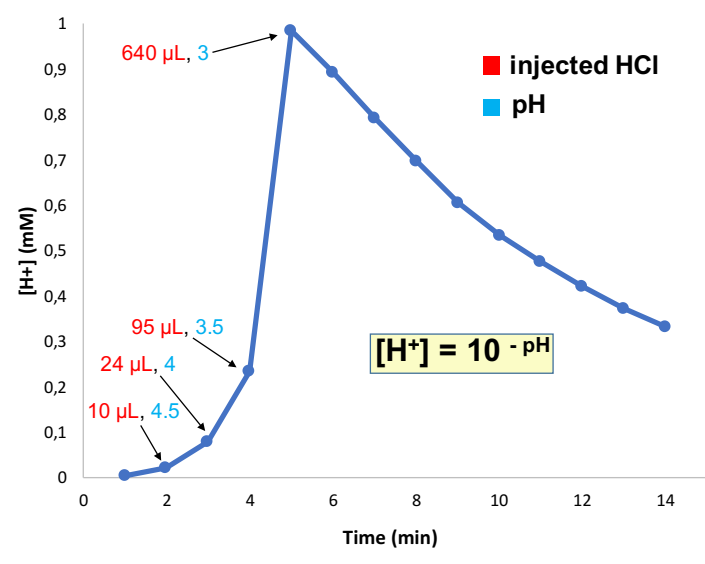

Fig. 4. pH test: measured concentrations and corresponding injected amounts.

2) $p H$ Level Test: With the second test we witnessed a change in $\mathrm{pH}$ (i.e. hydrogen ions concentration $\left[\mathrm{H}^{+}\right]$) in a given solution. The main aim of this test was to quantitatively evaluate the ability of the system to increase and decrease the concentration of a compound or ion. For this we used distilled water as neutral solution and $\mathrm{HCl} 0.01 \mathrm{M}$. A commercial $\mathrm{pH}$ meter was adopted to sense the $\mathrm{pH}$ variations. Therefore, $\mathrm{HCl}$ injections have been scheduled to achieve a $\mathrm{pH}$ decrease, corresponding to an increment in $\left[\mathrm{H}^{+}\right]$. We reproduced a preliminary phase characterised by fast ion concentration increase followed by an exponential decrease of $\left[H^{+}\right]$, trend in Fig. 4 . The test was performed in stirring condition (level 4 of the CB161 stirrer). Since we were mixing $\left[\mathrm{H}^{+}\right]$with distilled water, that contains $\left[\mathrm{H}^{+}\right]$itself (around $10^{-7} \mathrm{M}$ ), neither the dilution efficacy nor its accuracy can be assessed with this test.

3) APAP Concentration Test: Finally, we wanted to reproduce a trend similar to a real drug administration. To that aim, we adopted APAP as benchmark compound. The trend was obtained by injecting an APAP solution six times (one dose every 75 seconds) and by monitoring the dilution progress in the following 15 minutes, as shown in Fig. 5. The base-volume was $6 \mathrm{~mL}$ and the peristaltic flow was set at $0.86 \mathrm{~mL} / \mathrm{min}$. PBS solution was used as buffer solution. The base-volume solution was stirred between each measurement to ensure good mixing after drug injection (200 RPM VWE stirrer). To quantify APAP concentration in the base-volume solution CV measurements 


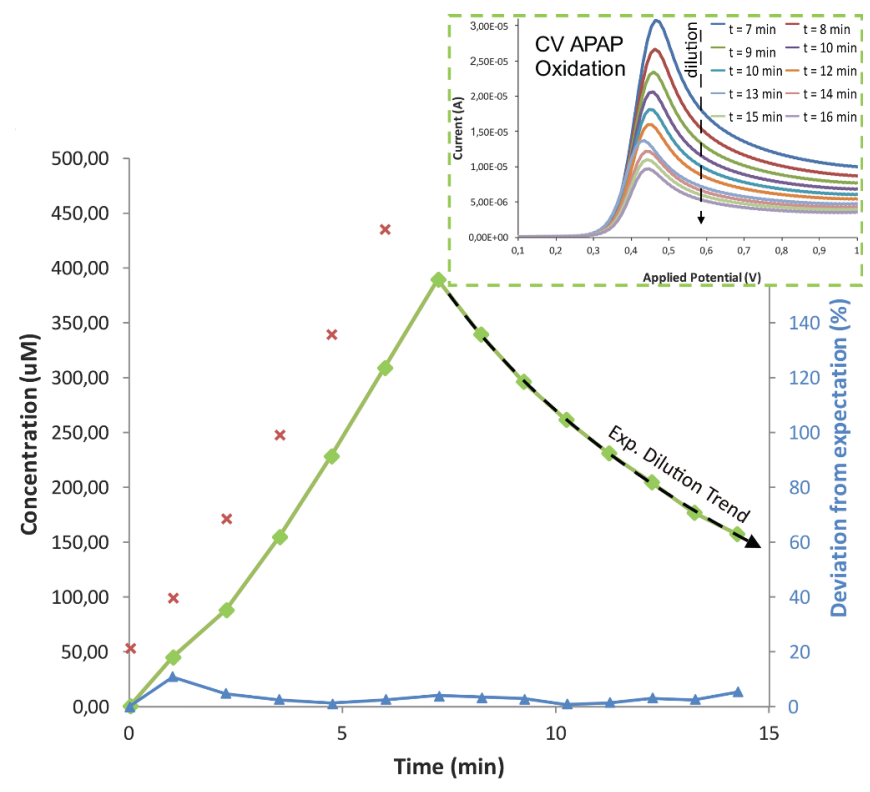

Fig. 5. APAP test: in the graph the sensed concentration for each measurement in time (green line), the evaluation in $\%$ of the variation between measured and expected concentrations (blue line) and the injected doses (red crosses). In detail the $\mathrm{CV}$ oxidation peaks for the dilution.

were performed.

Since APAP is directly measurable by $\mathrm{CV}$ technique, it was possible to identify the main trade-offs of the realised system. These are: (i) the dimension of the base-volume with respect to the syringe injection speed (evaluated from syringe dimensions), (ii) the constant speed of the peristaltic flow, (iii) the stirring condition needed to obtain a well-mixed solution.

\section{CONClusions}

The implementation and validation of a flow-injection system has been reported. The main aim of our system is to be adopted to facilitate and automate in-vitro testing of continuous monitoring systems for medical applications. Indeed, the administration of an effective drug dosage can be assessed only by applying a continuous monitoring system able to keep under control the concentration level of the drug in the patient. The characterisation of these monitoring devices is performed starting from in vitro experiments that are extremely laborious and time-consuming. In this context, our system wants to offer a faster and more reproducible way to test sensing platforms, e.g. electrochemical sensors.

The performance of the system has been verified by performing different tests. In this way it was possible to identify as main trade-offs of the system: (i) the dimension of the base-volume with respect to the syringe injection speed (evaluated from syringe dimensions), (ii) the constant speed of the peristaltic flow, and (iii) the importance of a stirring action in obtaining a well-mixed solution to sense. With the parameters we have set, we succeeded in reproducing the increasing and decreasing trend of a drug. The trend we obtained is similar to the human metabolic response when drugs are administered, e.g. during anaesthesia induction. Overall, the system is potentially able to reproduce any concentration trend within its intrinsic limits (e.g. tubing dimensions, base-volume, and injection syringe volume) depending on the chosen parameters. Further, the system can be applied not only to a base-volume contained in a beaker, but also to whatever fluidic unit containing the sensors platforms. Further improvement of the system may be obtained connecting a programmable peristaltic pump to the system. In this way, it could be possible to interrupt the dilution flow whenever static conditions are required for the measurement.

Future work will be the complete integration of this flow injection system within a feedback-loop system for anaesthesia monitoring [17].

\section{ACKNOWLEDGMENTS}

The authors thank A. Simalatsar and T. Kilic for helping in conceiving the system configuration, and I. Tzouvadaki for her writing tips. This work was supported by the CoMofA project (\#325230 157139), with grant from the Swiss National Science Foundation.

\section{REFERENCES}

[1] S. Anastasova, B. Crewther, P. Bembnowicz, V. Curto, H. M. Ip, B. Rosa, and G.-Z. Yang, "A wearable multisensing patch for continuous sweat monitoring," Biosensors and Bioelectronics, vol. 93, pp. 139-145, 2017.

[2] D. C. Klonoff, "Continuous glucose monitoring," Diabetes care, vol. 28, no. 5, pp. 1231-1239, 2005 .

[3] S. Carrara, A. Cavallini, V. Erokhin, and G. De Micheli, "Multi-panel drugs detection in human serum for personalized therapy," Biosensors and Bioelectronics, vol. 26, no. 9, pp. 3914-3919, 2011.

[4] J. Wang, "Amperometric biosensors for clinical and therapeutic drug monitoring: a review," Journal of pharmaceutical and biomedical analysis, vol. 19, no. 1, pp. 47-53, 1999.

[5] A. S. Gross, "Best practice in therapeutic drug monitoring," British journal of clinical pharmacology, vol. 46, no. 2, pp. 95-99, 1998

[6] F. Stradolini, T. Elboshra, A. Biscontini, G. De Micheli, and S. Carrara, "Simultaneous monitoring of anesthetics and therapeutic compounds with a portable multichannel potentiostat," in Circuits and Systems (ISCAS), 2016 IEEE International Symposium on. IEEE, 2016, pp. 834-837.

[7] B. Li, Z. Zhang, and W. Liu, "Flow-injection system for automated dissolution testing of isoniazid tablets with chemiluminescence detection," Talanta, vol. 54, no. 4, pp. 697-702, 2001.

[8] L. Z. Benet, D. Kroetz, L. Sheiner, J. Hardman, and L. Limbird, "Pharmacokinetics: the dynamics of drug absorption, distribution, metabolism, and elimination," Goodman and Gilman's the pharmacological basis of therapeutics, pp. 3-27, 1996.

[9] U. Yogeswaran and S.-M. Chen, "A review on the electrochemical sensors and biosensors composed of nanowires as sensing material," Sensors, vol. 8, no. 1, pp. 290-313, 2008.

[10] A. Simalatsar, M. Guidi, and T. Buclin, "Cascaded pid controller for anaesthesia delivery," in Engineering in Medicine and Biology Society (EMBC), 2016 IEEE 38th Annual International Conference of the. IEEE, 2016, pp. 533-536.

[11] F. Ortega, E. Dominguez, G. Jönsson-Pettersson, and L. Gorton, "Amperometric biosensor for the determination of phenolic compounds using a tyrosinase graphite electrode in a flow injection system," Journal of biotechnology, vol. 31, no. 3, pp. 289-300, 1993.

[12] X.-Z. Liu and Z.-L. Fang, "Sequential-injection system for drugdissolution studies of ibuprofen tablets and sustained-release formulations," Analytica chimica acta, vol. 358, no. 2, pp. 103-110, 1998.

[13] N. Wangfuengkanagul and O. Chailapakul, "Electrochemical analysis of acetaminophen using a boron-doped diamond thin film electrode applied to flow injection system," Journal of pharmaceutical and biomedical analysis, vol. 28, no. 5, pp. 841-847, 2002.

[14] B. S. Inc. ${ }^{\text {TM }}$, BS-8000/9000 Multi-Phaser Programmable Syringe Pump Manual.

[15] Gilson ${ }^{\mathrm{TM}}$, Minipuls 3 Peristaltic Pump User's Guide.

[16] E. W. Finucane, Definitions, Conversions, and Calculations for Occupational Safety and Health Professionals, Third Edition. CRC Press, 2017.

[17] F. Stradolini, A. Tuoheti, P. Motto Ros, D. Demarchi, and S. Carrara, "Raspberry pi based system for portable and simultaneous monitoring of anesthetics and therapeutic compounds," IEEE NGCAS, 2017. 